\title{
Mixed Reality Cane Simulation
}

\author{
D. Tzovaras ${ }^{1}$, K. Moustakas ${ }^{1,2}$, G. Nikolakis ${ }^{1}$, M.G. Strintzis ${ }^{1,2}$ \\ ${ }^{1}$ Informatics \& Telematics Institute \\ $1^{\text {st }} \mathrm{Km}$ Thermi-Panorama Road, PO Box 361 , \\ GR-57001 Thermi-Thessaloniki, Greece \\ \{tzovaras, moustak, gniko\}@iti.gr \\ ${ }^{2}$ Electrical and Computer Engineering Department \\ Aristotle University of Thessaloniki \\ GR-54006 Thessaloniki, Greece \\ strintzideng.auth.gr
}

\begin{abstract}
In the present paper a mixed reality cane simulation environment is presented that allows blind people to navigate in virtual worlds. The application is based on the combination of a real cane with a haptic force feedback system. The users can therefore handle the cane in a natural way and perceive realistic force feedback from virtual objects. Experimental results demonstrate the advantages of the MR approach when compared to the VR one.
\end{abstract}

\section{Introduction}

Virtual Reality (VR) and Mixed Reality (MR) systems are generally based on the use of advanced displays in order to provide an immersive visual interface. However VR and MR applications are not limited to visual feedback [1]. Audio feedback as well as haptic feedback can be used for the creation of immersive applications. These modalities are also suitable for the creation of applications for the blind and the visually impaired.

The HOMERE system presented in [2] is a multimodal system designed so as to aid the visually impaired people to explore and navigate inside virtual environments. The system provides the user with different sensations when navigating inside a virtual world: a force feedback corresponding to the manipulation of a virtual blind cane, a thermal feedback corresponding to the simulation of a virtual sun, and an auditory feedback in spatialized conditions corresponding to the ambient atmosphere and specific events in the simulation. A visual feedback of the scene is also provided to enable sighted people to follow the navigation of the main user.

Please use the following format when citing this chapter:

Tzovaras, Dimitrios, Moustakas, Konstantinos, Nikolakis, Georgios, Strintzis Michael, 2006, in IFIP International Federation for Information Processing, Volume204, Artificial Intelligence Applications and Innovations, eds. Maglogiannis, I., Karpouzis, K., Bramer, M., (Boston: Springer), pp. 353-360 
In $[3,4]$ a haptic virtual reality tool developed for the training of the visually impaired is presented. The proposed approach focuses on the development of a highly interactive and extensible Haptic Virtual Reality training system that allows visually impaired, to study and interact with various virtual objects in specially designed virtual environments, while allowing designers to produce and customize these configurations. The training scenarios include cane simulation, used for performing realistic navigation tasks.

The purpose of this paper is to develop a mixed reality cane simulation environment based on the existing VR cane simulation application $[3,4]$ and to conduct tests with blind users in order to obtain measurable results and derive qualitative and quantitative conclusions on the added value of the mixed reality system. The CyberGrasp haptic device was selected, based on its commercial availability and maturity of technology. In this paper we have developed a mixed reality environment for cane simulation and performed comparative tests with end users in order to identify the advantages of the mixed reality cane simulation. Moreover a novel superquadric based collision detection algorithm was integrated and tested with the system.

The paper is organized as follows. Section 2 describes an overview of the cane simulation system, including a general flow chart and features available in both the VR and MR systems. Section 3 describes the existing VR cane simulation system and including details of the hardware setup as well as information concerning the force feedback calculation. Section 4 describes the novel mixed reality cane simulation application. Section 5 describes the usability evaluation of the system. Finally, Section 6 draws the conclusions.

\section{System Overview}

This section describes the hardware setup used for the cane simulation applications (both VR and MR) as well as the software components that compose the cane simulation platform.

\subsection{Hardware setup}

The cane simulation hardware prototype consists of the CyberGrasp ${ }^{\mathrm{TM}}$ haptic device, a powerful workstation with specialized 3D graphics acceleration, input devices (primarily mouse and keyboard) and speakers (Figure 1).

The prototype handles both human-hand movement input and haptic forcefeedback using Immersion's CyberGlove(B) and CyberGrasp ${ }^{\mathrm{TM}}$ haptic devices [5]. CyberGlove ${ }^{\circledR}$ is a widely used human-hand motion-tracking device of proven quality. CyberGrasp ${ }^{\mathrm{TM}}$ is currently one of the very few force-feedback devices that are offered commercially, providing high quality of construction, operation and performance. The $350 \mathrm{~g}$ CyberGrasp ${ }^{\mathrm{TM}}$ exoskeleton is capable of applying a maximum of $12 \mathrm{~N}$ per finger force-feedback at interactive rates and with precise control. Both devices are supported by the VHSTM software developer kit, which allows straightforward integration with custom virtual reality software. 
An important component of the cane simulation system is the motion tracking hardware and software, required for tracking the position and orientation of the hand of the user. The system prototype utilizes Ascension's MotionStar Wireless ${ }^{\text {TM }}$ [6] motion tracker to accomplish this task. Other motion trackers, offering similar or better accuracy and responsiveness and a similar way of communication via local network, can easily be plugged into the system.

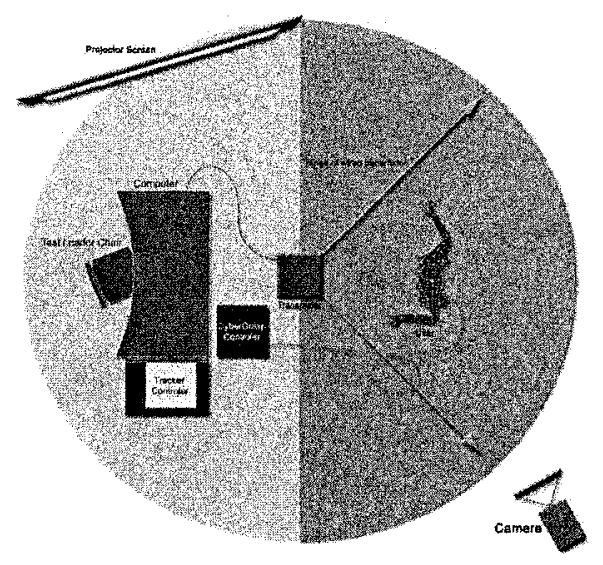

Fig 1. Cane Simulation Setup

\subsection{Application core}

The application consists of the following three main parts: a) initialization part, b) haptic loop and c) visual loop. The initialization part establishes connection with the hardware devices, reads the scene, initializes the collision detection algorithm and starts the haptic and visual loops. The haptic loop updates the scene using data from the devices, checks for collisions between the hand and scene objects, sets the new position of the hand and objects, triggers feedback forces and enables sound playback. The visual loop reads the current position of scene objects and renders the scene.

Figure 2 presents the general flow chart for the mixed reality cane simulation system. It is similar to the flow chart of the original VR application and includes the tracking of the real cane and a new module for force feedback calculation.

Collision detection is performed between the virtual cane and the VR scene using an SQ based collision detection algorithm presented in [7]. In order to check for collision between an object and the cane, the implicit formulae of the superquadrics is calculated for each point of the scene object. If the result is less than one at least for a point of the object, collision is reported.

If $F_{i}(x, y, z), 1 \forall S Q_{i}$; the point $(x, y, z)$ lies outside the cane.

else the point $(x, y, z)$ lies inside the cane.

The cost of executing collision detection tests only for the vertices composing a 3D mesh, and not for the elementary surfaces as most traditional collision detection 
methods do, is that in cases, where the mesh is coarse and the triangles are relatively large, the superquadric may penetrate a triangle or even pass through it without collision report. In order to solve this problem additional control points are inserted inside each triangle so that the distance between adjacent points is constant, i.e. a $2 \mathrm{D}$ grid is applied onto the triangle. In this way the collision tests are performed for the control points for each segment of the virtual hand.

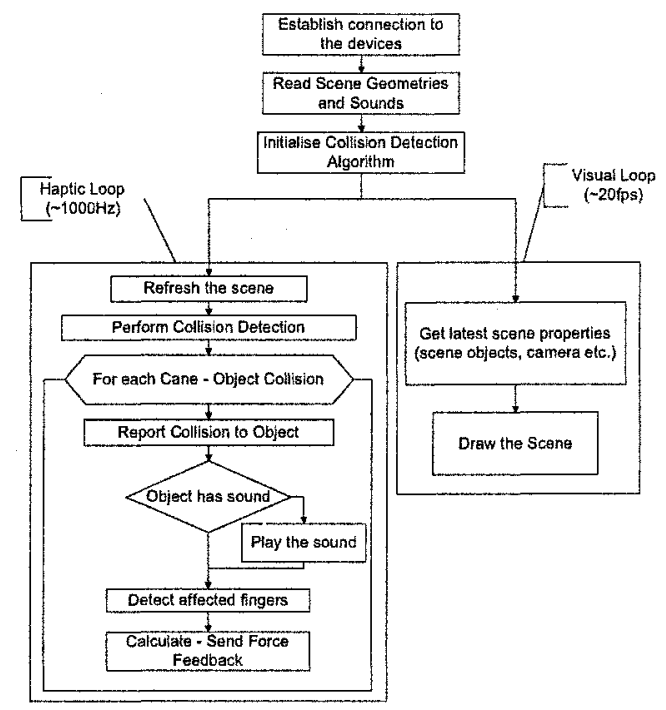

Fig 2. General flow chart of the cane simulation environment

The system supports also 3D sound using the OpenAL (www.openal.org)library in order to create realistic audio feedback.

\section{VR Cane Simulation}

Cane simulation, has been used for performing realistic navigation tasks with the use of CyberGrasp, which in combination with the Ascension MotionStar wireless tracker leads to a significant workspace (up to 7 meters). These simulations include indoor and outdoor environments such as navigation in the interior of a bank or a public building, traffic light crossing, etc.

The cane was simulated to be an "extension" of the users index finger. The relative position between the index finger and the top of the cane is illustrated in Figure 3.

The force feedback applied to the hand of the user depends on the collision of the cane and the scene objects. When the cane hits on the ground, force feedback is send to the index finger of the user (shown in Figure 3). Force feedback is applied to the 
thumb when the cane collides with an object laying on its right side (shown in Figure 3 ). Force feedback is applied to the middle ring and pinky fingers when the cane collides with an object being on its left side (Figure 3).
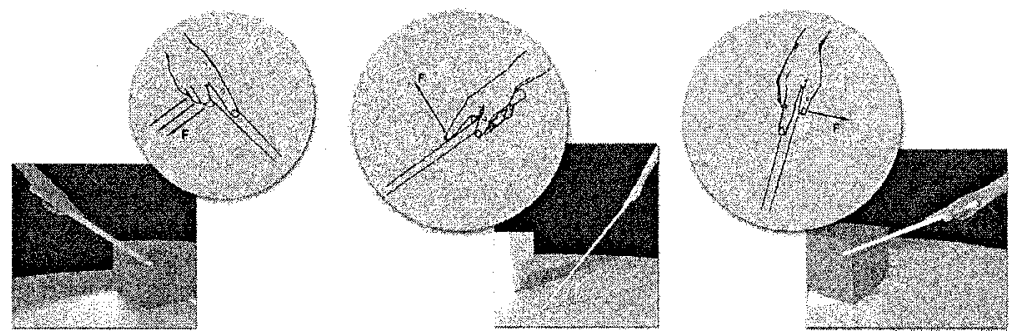

Fig. 4. Cane collision with the ground, an object on the left hand side of the user and an object on the right hand side of the user.

The forces applied to the user are: a constant continuous force that emulates the force provided by grasping a real cane, a cosine force effect (buzzing) provided to the user when the cane is penetrating an object and a Jolt force effect is send to the user when the cane hits an object or the ground. Cosine force effect is described by the equation

$$
\mathrm{F}=\mathrm{a}(1+\cos (2 \pi \omega \mathrm{t}))(1)
$$

the Jolt force effect by the equation

$$
\mathrm{F}=\mathrm{ae} \mathrm{e}^{-\mathrm{kt}} \text {. }
$$

where, $\mathrm{a}$ is the amplitude of the force and $\mathrm{k}$ is the attenuation factor. The cane simulation application is adjustable in terms of:

- the cane length,

- the grasping force, the floor hit force and the wall hit force and

- the level of buzzing (force when cane is penetrating an object),

in order to make the test leader able to modify the simulation parameters online, based on the user requirements.

\section{MR Cane Simulation}

The MR interface is an extension of the interface described in the VR cane simulation application. The user wears the CyberGrasp and a waistcoat for carrying the Force Control Unit (FCU) for the CyberGrasp and the Motionstar control unit to connect the magnetic sensors. The first sensor is attached to CyberGrasp device and the second sensor is attached to the real white cane. Sound and haptic feedback are provided by the system upon collision of the cane with the virtual objects with 
respect to the position of the objects in the scene and the orientation and position of the users head. The parameters of the virtual cane (size, collision forces) are adjusted so that it fits to the real cane and the user can perceive the contacts similarly as with the real one. Environmental sounds are assigned to static objects in the scene (e.g. realistic traffic lights sound is assigned to traffic lights in the virtual scene) as well as to dynamic objects (e.g. cars).
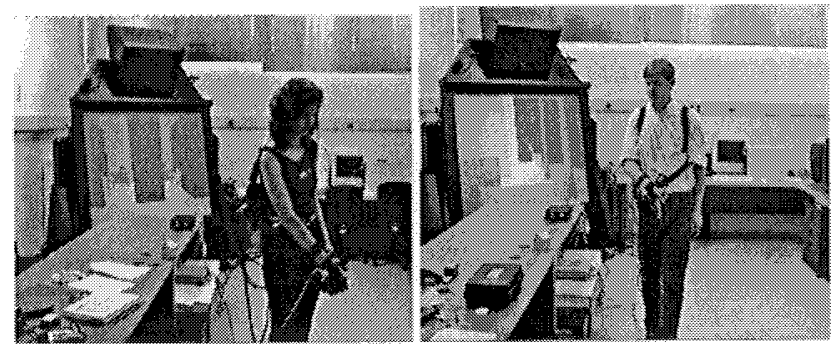

Fig. 4. Users practicing in the cane simulation environment

Force Feedback calculation in the case of the MR application is more complex than in the VR case. The grasping force is deactivated since the user grasps the real cane. A simplified dynamics model is used to calculate the force feedback applied to the fingers. The proposed model takes into account that Cybergrasp can apply forces approximately perpendicular to the users fingertips. In order to detect fingers that should perceive force the relative position of the user's digits and the cane is calculated along side with the moving direction of the cane. Force effect amplitude is multiplied by the cosine of the angle between the cane direction vector and a vector perpendicular to the finger digit (Figure 5).

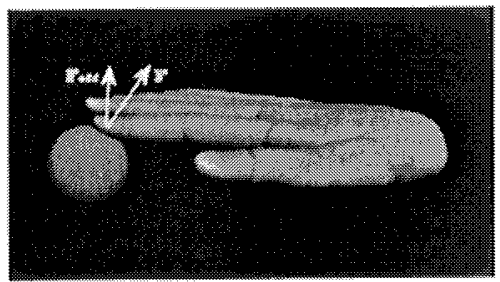

Fig. 5. Force feedback for the CyberGrasp

The main advantage of the MR cane simulation over the VR system is that the user can handle the cane as in real world conditions without any restrictions in the grasping way. This cannot be implemented in the VR system because grasping an object (i.e. the cane) in the desired way without using any visual feedback is a difficult task and could cause inconsistence between the actual position of the VR cane and the position imagined by the user. 


\section{Usability Evaluation}

Initial versions of the applications have been evaluated with blind and visually impaired users. Specifically, the white cane simulation has been tested with blind and visually impaired users from the Thessaloniki Blind School and the Pan-Hellenic Blind association.

Twenty-six persons participated in the tests from the Central Macedonia Local Union of the Panhellenic Association for the Blind in Greece. The users were selected so as to represent the following groups: blind from birth, blind at a later age, adults, and children. The evaluation consisted of three phases. In the first phase the users were introduced to the system and were allowed to use it for a while in order to get used to the device and to calculate the most comfortable parameters for the cane (i.e. length, force amplitude).

In the second phase the users performed the tasks. The total time to complete the task, users comments and success or failure in performing the task were recorded for each user. In the third face the users answered questionnaire, about the performance and the usability of the system.

According to the comments of the users during the tests and their response to the questionnaires, the following conclusions can be drawn: It was deemed very important to utilize both acoustic and haptic feedback, as they are indispensable for the orientation. It is also important to note that a percentage ranging from $96 \%$ of the users have characterized the tests as useful or very useful.

An initial comparative test has been performed to estimate the importance of using a mixed reality system over a virtual reality system as well as the significance of each modality to users navigation. Specifically, the system was evaluated for the following cases

- MR with haptic feedback, without audio feedback

- MR without haptic feedback, with audio feedback

- VR with multimodal feedback (both haptic and audio)

- MR with multimodal feedback.

The evaluation was based on psychophysical criteria and was performed using questionnaires. The results showed that the users preferred in terms of usability the MR simulation with multimodal feedback. The second choice was the VR with multimodal feedback. Third was the MR using only audio feedback and last was the MR using only haptic feedback. The ranking shows the importance of the multimodal feedback.

\section{Conclusions}

The evaluation results on the initial version of the system were promising. The fact that blind persons use different ways to grasp the cane led to the decision of creating an application that can simulate various ways of grasping and using the cane. This led to the design of the MR cane simulation system, which tracks the position of the users hand and the real white cane. 
In the cases of the cane simulation, technical limitations constrain its applicability. Specifically, the system cannot prevent the user from penetrating objects in the virtual environment. The maximum workspace is limited to a $7 \mathrm{~m}$ diameter hemisphere around the tracker transmitter (the $1 \mathrm{~m}$ limitation, caused by the CyberGrasp ${ }^{\mathrm{TM}}$ device is solved by using a backpack so that the user can carry the CyberGrasp ${ }^{\mathrm{TM}}$ actuator enclosure). The maximum force that can be applied is limited to $12 \mathrm{~N}$ per finger and the feedback update rate is $1 \mathrm{KHz}$.

Concluding, the usability evaluation results demonstrate that the proposed mixed reality application was considered as an improvement of the original work $[3,4]$, whereas it still leaves a lot of room for improvement and supplement. Provided that further development is carried out, the system has the fundamental characteristics and capabilities to incorporate many requests of the users for the creation of a more realistic training environment.

The approach chosen, fully describes the belief of blind people to facilitate and improve training practices. It represents an improvement of life for the blind and the visually impaired people when connected to reality training. These facts are evident from the participants' statements.

Except from the direct benefits of the proposed system, as many of the users mentioned, the technology based on virtual environments can eventually provide new training and job opportunities to people with visual disabilities.

Acknowledgment This work has been conducted in conjunction with the "SIMILAR" European Network of Excellence on Multimodal Interfaces of the IST Programme of the European Union (www.similar.cc).

\section{References}

1. Burdea G.C. and Coiffet P. Virtual Reality Technology (2nd ed.). Wiley-IEEE Press (2003).

2. Lecuyer A., Mobuchon P., Megard Ch., Perret J., Andriot C., Colinot J.P.: HOMERE: a Multimodal System for Visually Impaired People to Explore Virtual Environments, IEEE Virtual Reality Conference 2003 (VR'03), (2003) 251.

3. D. Tzovaras, G. Nikolakis, G. Fergadis, S. Malasiotis and M. Stavrakis.: Design and Implementation of Haptic Virtual Environments for the Training of Visually Impaired, IEEE Trans. on Neural Systems and Rehabilitation Engineering, Vol. 12, No. 2, (2004) 266-278.

4. D. Tzovaras, G. Nikolakis, G. Fergadis, S. Malassiotis and M. Stavrakis: Virtual Environments for the Training of Visually Impaired, CUWATTS Conference, Cambridge, (2002).

5. Immersion Technologies Inc.: Virtual Hand Suite 2000: User \& Programmer Guides, http://www.immersion.com/3d/products/virtualhand_sdk.php.

6. Ascension Technology Corporation: MotionStar Wireless, http://www.ascensiontech.com/.

7. K. Moustakas, G. Nikolakis, D. Tzovaras and M.G. Strintzis, "Haptic Feedback Using an Efficient Superquadric Based Collision Detection Method", in First Joint Eurohaptics Conference and Symposium on Haptic Interfaces for Virtual Environment and Teleoperator Systems, Pisa (2005). 